\section{Health-Info-Net: une chance à saisir!}

\author{
F.-X. Deschenaux ${ }^{1}$, G. von Below ${ }^{2}$ \\ ${ }^{1}$ Président du conseil d'administration \\ ${ }^{2}$ Directeur de Health-Info-Net SA
}

Il y a 3 ans, la FMH a créé le Health-Info-Net (HIN), un réseau internet à la pointe de la technique, répondant à des normes de sécurité internationalement reconnues. Aujourd'hui, ce sont plus de 2000 médecins qui profitent déjà, dans leur activité quotidienne, des nombreuses et intéressantes possibilités d'utilisation de l'internet offertes par le HIN.

\section{Pourquoi la FMH a-t-elle créé le HIN?}

Les principales raisons de la création de ce réseau centré sur les besoins particuliers des professions médicales étaient les suivantes:

Accès aux connaissances et aux faits Dans le HIN, les médecins trouvent rapidement et sans difficulté des données et des faits se rapportant aux travaux et projets scientifiques, ainsi que des informations sur les hôpitaux, les entreprises et d'autres groupements dans le domaine de la santé publique. Le HIN se veut un moyen par lequel les médecins et leurs partenaires du domaine de la santé peuvent échanger des informations et entretenir des relations d'affaires.

\section{Sécurité sur l'internet}

Vos données sont protégées contre tout accès non autorisé! Les technologies de cryptage et de contrôle d'accès utilisées vous permettent d'échanger des données sensibles en toute sécurité avec d'autres membres du réseau HIN, que ce soit par le biais du courrier électronique ou dans un forum de discussion sécurisé. De plus, si vous optez pour le «HIN-PowerProviding", votre ordinateur sera à l'abri du piratage informatique.
Information et assistance technique

Un abonnement HIN permet, au besoin, de bénéficier, via une ligne d'assistance technique, de l'aide compétente et du soutien d'une équipe expérimentée et qualifiée. Celle-ci se tient à la disposition des utilisateurs et leur fournit des conseils utiles et des services valables à des prix raisonnables.

\section{Le phénomène internet prend l'ampleur!}

Les spécialistes de la branche estiment que, d'ici la fin de l'année, un ménage sur trois, voire sur deux, disposera d'un raccordement à l'internet. Aujourd'hui, dans notre pays, 35\% des médecins y sont connectés et, parmi eux, les 2000 abonnés du HIN. Pour une taxe annuelle de Fr. 180.-, ces derniers profitent de toute une série de prestations et de droits d'accès ainsi que d'un espace sécurisé selon les plus hautes exigences techniques pour l'échange de données sensibles (ayant trait à leurs patients) via l'internet.

Pourtant, de nombreux membres de la FMH doutent aujourd'hui encore des avantages de l'internet et du HIN. Pour cette raison - ou pour une autre - ils ne se sont pas encore décidés pour un raccordement:

- Nombre d'entre eux ne voient pas pourquoi il faudrait forcément entrer dans l'âge de l'électronique.

- Certains émettent des doutes quant au rapport coûts/utilité; pour eux, il y a finalement suffisamment de fournisseurs proposant une offre intéressante avec un accès gratuit - mais justement non protégé - à l'internet.

- Il n'y a pas eu de contrainte extérieure jusqu'ici; la technologie moderne a de plus un effet désécurisant: ce bon vieux papier n'a-t-il pas finalement toujours fait l'affaire?

- Des craintes parfaitement compréhensibles sont également présentes concernant la protection des données et les abus possibles en la matière. Pourtant, qui, quelle institution, pourrait, le cas échéant, avoir un accès non autorisé aux données du cabinet médical, de l'hôpital ou même du corps médical, si les fournisseurs de prestations disposent d'un réseau pour leurs échanges? Certes, les évènements rapportés par la presse concernant des virus tels que Melissa ou ILOVEYOU, qui ont causé pour des milliards de francs de dégâts dans le monde entier, ne contribuent pas à dissiper les doutes et les craintes sur l'internet. Mais, il ne s'agit pas là de problèmes spécifiques au HIN; tous les fournisseurs d'accès sont en effet concernés dans la même mesure. Cela dit, pour ce qui est de la protection des données, il est tout à fait justifié d'avoir quelque inquiétude lorsqu'on dispose d'une connexion normale à l'internet et d'un fournisseur commercial pour son courrier électronique au lieu du HIN.
Correspondance:

Georg von Below

Health-Info-Net AG

Zürichstrasse 64

CH-8700 Küsnacht 


\section{Pourquoi un abonnement au HIN pour tous les membres de la FMH?}

La phase de mise en route étant terminée, la société HIN - dont la FMH est l'actionnaire majoritaire - souhaite changer de système de financement, en passant d'un réseau financé en majeure partie par des abonnements individuels à une structure assurant ellemême son financement via les offres de prestataires commerciaux. Aujourd'hui déjà, dans le marché en rapide mutation de l'e-business, la société HIN offre une infrastructure idéale au prestataire de service souhaitant communiquer avec des médecins établis. Qu'il s'agisse d'hôpitaux, de laboratoires, de fournisseurs de médicaments ou de matériel de consommation, tous apprécient les avantages d'un réseau sécurisé au sein duquel les partenaires commerciaux sont identifiés et leurs messages munis d'une signature électronique. C'est en effet la seule manière de créer des relations d'affaires sûres via l'internet.

Toutefois, pour les divers prestataires, le HIN ne présente un intérêt en tant que réseau qu'à deux conditions:

1. Les normes technologiques doivent répondre aux exigences particulières de la protection des données dans le domaine de la santé publique.

$\Rightarrow$ La technologie ASAS utilisée sur le HIN remplit cette condition; certains experts la qualifient de technologie de pointe. Les banques et les hôpitaux ont d'ailleurs commencé à s'en servir.

2. Les prestataires en question doivent pouvoir, le plus rapidement possible, s'adresser à la majeure partie des membres FMH - voire tous! - par le biais du HIN. Tout comme le Bulletin des médecins suisses qui est envoyé à chaque membre de la FMH, le HIN doit jouer le rôle de canal d'information pour le corps médical.

Si ces conditions ne sont pas remplies, le HIN ne sera pas d'un intérêt suffisant pour les prestataires potentiels disposant de moyens financiers confortables et, ce qui risque de rendre les échanges d'informations plus compliqués et peu pratiques à l'avenir, de nombreux prestataires développeront leur propre système de sécurité.

$\Rightarrow$ A cet égard, le HIN offre une technologie uniforme, élégante et polyvalente, qui satisfait à toutes exigences nécessaires pour établir des normes technologiques de sécurité dans le domaine de la santé publique.

\section{Proposition d'abonnement au HIN pour tous les membres de la FMH}

Pour atteindre ce but, le Comité central de la FMH a décidé de présenter à la Chambre médicale, lors de la séance des 21 et 22 juin, la proposition suivante:

1. Pour promouvoir l'utilisation d'internet par tous les médecins de Suisse, chaque membre de la FMH doit recevoir l'abonnement actuel au HIN gratuitement.
2. Le financement de cette action limitée à trois ans doit être assuré par une contribution spécifique de Fr. 50.- par année.

\section{Quels sont les avantages d'une telle démarche?}

Ceux qui sont déjà abonnés au HIN obtiendront les avantages suivants:

- Les coûts de l'abonnement annuel seront considérablement réduits et pourront être réglés avec le paiement de la cotisation FMH.

- L'effet promotionnel recherché encouragera de nouveaux confrères à profiter de la technologie internet. Le nombre d'interlocuteurs avec lesquels il sera possible d'échanger des informations sensibles ne cessera de croître.

- L'espace HIN deviendra une "place de marché» intéressante pour les autres partenaires de la santé publique ainsi que pour les prestataires de services: ils pourront y proposer de nouveaux produits et de nouvelles offres aux abonnés du HIN.

- Pour les médecins qui ne sont pas encore familiarisés avec l'internet et le HIN, ce sera l'occasion de faire connaissance avec cette nouvelle technologie.

- L'abonnement au HIN coûtera désormais bien moins cher et sera payé avec la cotisation FMH. Il serait dommage de ne pas saisir cette occasion!

- L'entrée dans l'âge de l'internet sera accompagnée d'actions spéciales pour l'achat d'ordinateurs portables "Plug \& Surf» (brancher et naviguer sur le net!), avec un accès à l'internet déjà installé; en outre, des cours pour débutants seront organisés.

- Vous pourrez découvrir progressivement les multiples possibilités de l'internet avant de vous en servir pour des applications commerciales.

\section{Une occasion à saisir!}

Le HIN est devenu un réseau d'importance nationale et même internationale. La communication et le commerce électroniques gagnent du terrain et le domaine de la santé ne saurait être épargné. Le temps est donc venu pour les médecins d'adopter des normes communes avant que cette possibilité ne leur échappe.

Le CC compte sur votre soutien à la prochaine séance de la Chambre médicale, les 21 et 22 juin 2000. Ne laissons pas passer cette chance! 